\title{
Measurement and correction of two-sided freeform optical elements with combined tactile-optical metrology equipment
}

\author{
Johannes Hartung ${ }^{1, *}$, Henrik von Lukowicz ${ }^{1,2}$, Mathias Rohde $^{1}$, Knut Kleinbauer ${ }^{1}$, Nils Heidler ${ }^{1}$, and Stefan Risse $^{1}$ \\ ${ }^{1}$ Fraunhofer Institute for Applied Optics and Precision Engineering IOF, Albert-Einstein-Strasse 7, 07745 Jena, Germany \\ ${ }^{2}$ Institute for Applied Physics IAP, Friedrich-Schiller-University Jena, Max-Wien-Platz 1, 07743 Jena, Germany
}

\begin{abstract}
Freeform optical elements are state of the art for several years to fabricate very high performance optical systems with the necessity of, e.g., strong folding in mirror system or correction of typical asymmetric aberrations in mirror systems as well as lens systems. For freeform mirror systems, in particular for metal mirrors, the metrology is well understood and iterative process chains are well established. For transmission elements with a freeform surface on both sides, manufacturing, metrology, and correction for both sides in a parallel manner is quite difficult. The article presents a method to measure such an optical element and correct it with a well-defined correction step to have both sides in a well-defined position to each other.
\end{abstract}

\section{Introduction}

Freeform optics are state of the art for several years now. They are often used in systems where compactness or the correction of asymmetric aberrations is important. Since freeform surfaces are more difficult in manufacturing and measurement, they are more expensive and therefore usually only used for high performance optics. For freeform metal mirrors a fabrication, metrology, and correction process chain is well-established from MIR to VIS wavelengths. The advantage of metal mirrors is that it is possible to manufacture references directly on the mirror substrate near the optical surface and that in mirror systems chromatic aberrations play no role at all $[1,2]$. For freeform lenses of high performance transmission optics, the situation is more complicated than for metal mirrors. First of all it is not possible to manufacture metrology references directly on the lens substrate. Further, lenses have usually not enough edge area to be usable for references. The most difficult part for manufacturing of freeform lenses is that if every side of the lens is a freeform, it is necessary to measure the two sides relative to each other to quantify the optical error introduced by the misalignment. In a second step, these errors have to be corrected while it is necessary to separate form and position error in a well-defined way. The article introduces a metrology equipment to make the measurement of form and position error possible by using different metrology methods and to later correct both sides of a freeform lens with respect to form and position.

\section{Preliminaries}

A lens is divided into two optical surfaces and an outer boundary. The two surfaces in the present case are freeforms from the viewpoint of manufacturing. This means there are more than two independent axes for machining necessary to manufacture such surfaces. The outer boundary consists of mounting structures and reference features for later measurement or mounting within a frame structure. For glassy elements it is difficult to manufacture reference spheres into the outer boundary. The only reference features available are angular flats, plane surfaces, and circular structures for centering (see, e.g., fig. 1).

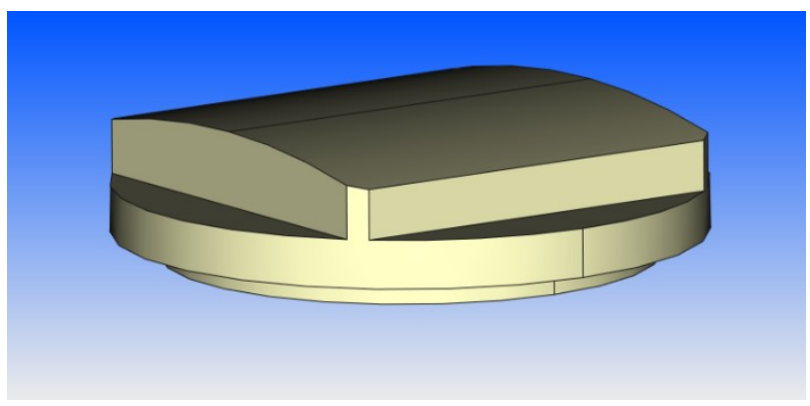

Fig. 1 Plano surface for alignment and circular boundary for centering. The plano surfaces in the XY plane can be used for detilt.

The overall challenge is to measure the reference structures at the boundary with respect to the optical surfaces to have some geometrical features as a first part in the measurement chain. From these measurements, it is possible to measure the positions of the optical surfaces

\footnotetext{
* Corresponding Johannes Hartung: johannes.hartung@iof.fraunhofer.de
} 
and separate form and position error from each other. The available measurement machines, which provide the necessary high measurement accuracy, are a coordinate measurement machine (Zeiss Mikura), a 2.5D profilometer (Panasonic UA3P), and an interferometer (Zygo DynaFiz). Since there is no possibility to get a common reference system for all three measurement machines, it is necessary to develop a measurement equipment (see fig. 2).

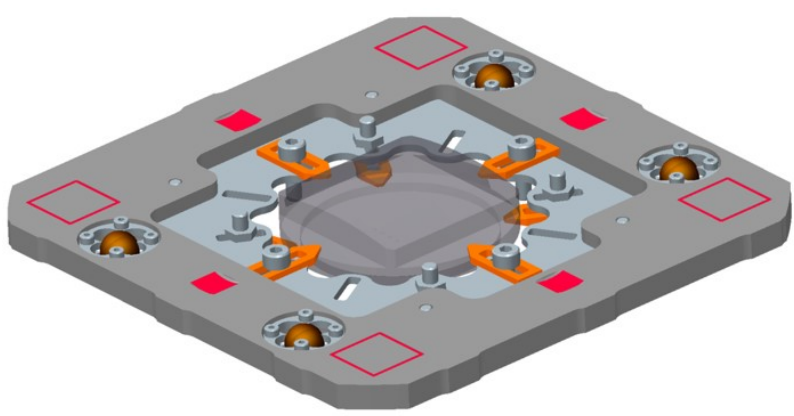

Fig. 2 Measurement equipment - CAD design. Red are the surfaces for alignment in different measurement machines. Dark yellow are the Rubin spheres. In the middle there is the inlay with a demonstrator optics for the project.

This equipment [3] is designed as a kind of frame which has several tactile and optical references on its exterior structure and a CNC machined inlay for fixing the lens itself. The interior inlay can be easily rebuilt for another lens whereas the outer structure needs to be designed for a certain size and accuracy of the measurements. The different reference structures are:

- 4 plano surfaces on each side for removing tip/tilt in front of an interferometer

- 4 cylindrical CGH references for fixing the position of the $\mathrm{CGH}$ in relation to the measurement equipment on each side

- 4 Rubin spheres built into the frame which are useful for finding a common coordinate systems for both sides

The final goal is to arrange all measurement data into one common coordinate system which leads to a high data analysis effort. But afterwards it is possible to perform a post-polish process for glassy materials with the help of Magnetorheological finishing (MRF). The relative position and the form of each of the freeform surfaces can therefore be generated with a high accuracy depending on the quality of the reference features.

For the MRF polishing part, it is necessary to manufacture another manufacturing equipment to fix the freeform surfaces with all six degrees of freedom such that the polishing indeed corrects for the positional as well as the form errors of the optical freeform element. This is necessary since the measurement equipment cannot be adapted for the integration into the MRF machine.

\section{Results}

Within the project the first data was generated by measuring a lens which has one plano side and another convex cylinder side with curvature radius of $40 \mathrm{~mm}$ along the $\mathrm{X}$ axis.

For this test part it was verified that it is possible to arrange profilometer as well as coordinate measurement data into one coordinate system from which it is possible to achieve a form and position correction onto that substrate (see fig. 3).
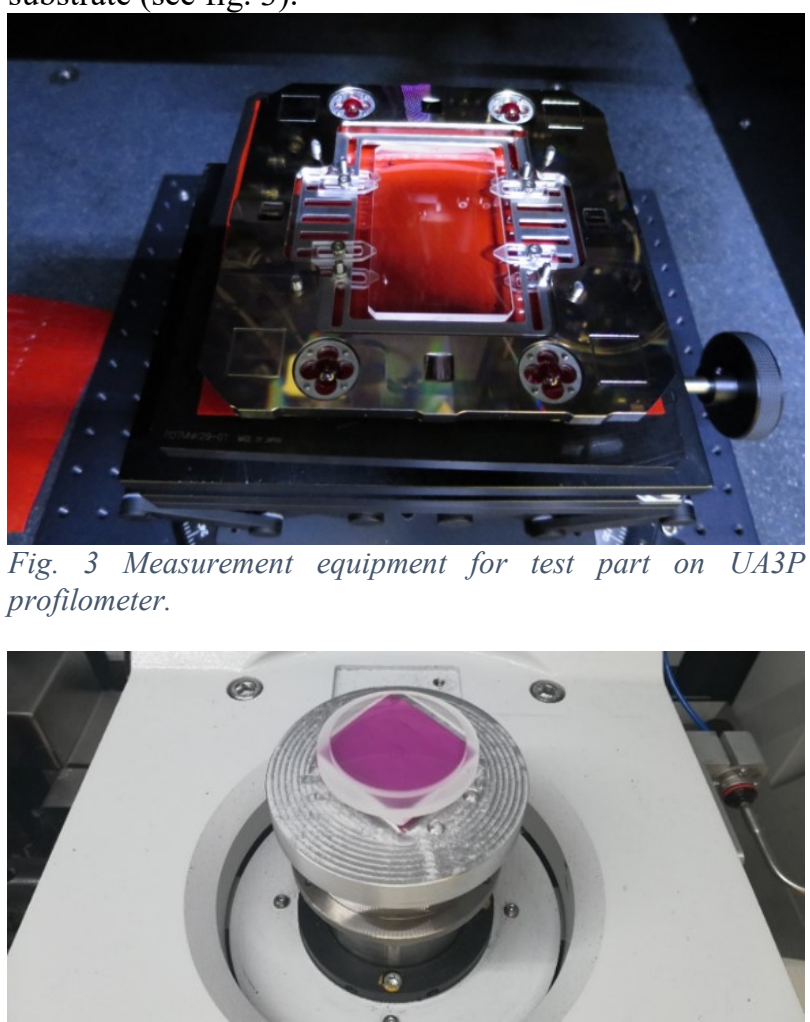

Fig. 4 Demonstrator element on MRF for form correction.

Additionally, an interface for the MRF correction of the demonstrator was manufactured (see fig. 4) to verify the positioning step using reference elements.

The authors would like to thank Matthias Beier for enlighting discussions, Wilko Fuhlrott for diamond turning of the measurement equipment, and Stephanie Hesse-Ertelt for language corrections. The research is funded in project Free4Optics under grant number 2015 FE 9148 by the Thüringer Aufbaubank (TAB) within the European Regional Development Fund (ERDF).

\section{References}

1. S. Scheiding, C. Damm, W. Holota et al., Proc. SPIE 7739, 773908 (2010)

2. M. Beier, J. Hartung, T. Peschel et al., Appl. Optics 54, 3530 (2015)

3. Patent 2018 F61223, Halterung zur Fixierung und Referenzierung von mit einer Messvorrichtung an mehreren, nicht in einer gemeinsamen Ebene angeordneten Oberflächen in einer Einspannung zu vermessenden Bauteilen 